\title{
Guanidine hydrochloride-induced reversible unfolding of sheep liver serine hydroxymethyltransferase
}

\author{
B Venkatesha, Jayant B Udgaonkar*, N Appaji Rao and H S Savithri ${ }^{\dagger}$ \\ Department of Biochemistry, Indian Institute of Science, Bangalore 560012, India \\ "National Centre for Biological Sciences, TIFR Centre, Indian Institute of Science Campus, PO Box 1234. \\ Bangalore 560012 , India \\ ${ }^{\dagger}$ Corresponding author (Fax, 91-80-3341683; Email, bchss@biochem.üisc.ernet.in).
}

Equilibrium unfolding studies of sheep liver tetrameric serine hydroxymethyltransferase (SHMT, EC 2.1.2.1) revealed that the enzyme assumed apparent random coil structure above $3 \mathrm{M}$ guanidine hydrochloride $(\mathrm{GdnHCl})$. In the presence of non-ionic detergent Brij-35 and polyethylene glycol, the $6 \mathrm{M} \mathrm{GdnHCl}$ unfolded enzyme could be completely ( $>95 \%$ ) refolded by a 40 -fold dilution. The refolded enzyme was fully active and had kinetic constants similar to the native enzyme. The midpoint of inactivation $(0.12 \mathrm{M} \mathrm{GdnHCl})$ was well below the midpoint of unfolding $(1-6 \pm 0-1 \mathrm{M} \mathrm{GdnHCl})$ as monitored by far UV CD at $222 \mathrm{~nm}$. In the presence of PLP, the midpoint of inactivation shifted to a higher concentration of $\mathrm{GdnHCl}(0-6 \mathrm{M})$ showing that PLP stabilizes the quaternary structure of the enzyme. However, $50 \%$ release of pyridoxal-5'-phosphate (PLP) from the active site occurred at a concentration $(0-6 \mathrm{M})$ higher than the midpoint of inactivation suggesting that $\mathrm{GdnHCl}$ may also act as a competitive inhibitor of the enzyme at low concentrations which was confirmed by activity measurements. PLP was not required for the initiation of refolding and inactive tetramers were the end products of refolding which could be converted to active tetramers upon the addition of PLP. Size exclusion chromatography of the apoenzyme showed that the tetramer unfolds via the intermediate formation of dimers. Low concentrations $(0-3-0-6 \mathrm{M})$ of $\mathrm{GdnHCl}$ stabilized at least one intermediate which was in slow equilibrium with the dimer. The binding of ANS was maximum at $0.4-0.6 \mathrm{M} \mathrm{GdnHCl}$ suggesting that the unfolding intermediate that accumulates at this concentration is less compact than the native enzyme. 